\title{
Effectiveness of Virtual Reality Eyeglasses as a Distraction Aid to Reduce Anxiety among 6-10-year-old Children Undergoing Dental Extraction Procedure
}

\author{
Paloni Koticha ${ }^{1}$, Farhin Katge ${ }^{2}$, Shilpa Shetty ${ }^{3}$, Devendra P Patil ${ }^{4}$
}

\begin{abstract}
Introduction: Distraction is commonly used nonpharmacologic pain management technique by pedodontists to manage pain and anxiety. There are some new techniques which uses audiovideo stimulation and distract the patient by exposing him or her to three-dimensional videos. These techniques are referred to as virtual reality audiovisual systems. The aim is to evaluate the effectiveness of virtual reality eyeglasses as a distraction aid to reduce anxiety of children undergoing extraction procedure.

Objective: The aim of this study is to evaluate the effectiveness of virtual reality eyeglasses as a distraction aid to reduce anxiety of children undergoing dental extraction procedure.

Materials and methods: Thirty children of age 6-10 years $(n=60)$ with bilateral carious primary molars indicated for extraction were randomly selected and divided into two groups of 30 each. The first one was group I (VR group) ( $n=30)$ and group II (control group) ( $n=30)$. Anxiety was measured by using Venham's picture test, pulse rate and oxygen saturation. Anxiety level between group I and group II was assessed using paired " $t$ " test.

Results: The mean pulse rate values after extraction procedure in group I were $107.833 \pm 1.356$ and group II were $108.4 \pm 0.927$ respectively. The pulse rate values in intergroup comparison were found statistically significant $p=0.03$.

Conclusion: The virtual reality used as a distraction technique improves the physiologic parameters of children aged 6-10 years but does not reduce the patient's self-reported anxiety according to Venham's picture test used.
\end{abstract}

Keywords: Audiovisual, Distraction, Oxygen saturation, Pulse rate, Venham picture test, Virtual reality eyeglasses.

International Journal of Clinical Pediatric Dentistry (2019): 10.5005/jp-journals-10005-1640

\section{INTRODUCTION}

Dental anxiety is defined as "an abnormal fear or dread of visiting the dentist for preventive care or therapy and unwarranted anxiety over dental procedures."1 It may have psychological, cognitive, and behavioral consequences. It exists in considerable proportion of children making them uncooperative during dental visits. The presence of anxiety in children suggests that the nervous system is vulnerable to noxious stimuli during development. Alleviating child's anxiety toward dental treatment is important not only in mitigating immediate fear but also in preventing apprehension continuing into adulthood. ${ }^{2}$

Management strategies have been proposed to reduce distress during dental treatment in children and are mainly divided into two broad categories. The first module consists of behavioral techniques including the tell-show-do technique, distraction, inspiration, modeling, and hypnotism. The second category consists of pharmacologic techniques. ${ }^{3}$ Among these, the distraction technique is found to be effective among children in reducing anxiety. ${ }^{4}$

Distraction is a useful technique of diverting the patient's attention from what may be perceived as an unpleasant procedure. This enables decreased perception of unpleasantness and averting negative behavior. Kleiber and Harper suggested distraction as a cognitive coping mechanism that passively redirects the subject's attention or actively involves the subject in the task. ${ }^{5}$ Giving the patient a short break during a stressful procedure can be an effective use of the distraction technique prior to considering more advanced behavior guidance techniques. Recent dental studies have shown that distraction is a common technique used to reduce the pain reaction during short invasive procedures. ${ }^{6-10}$
${ }^{1-4}$ Department of Pedodontics and Preventive Dentistry, Terna Dental College, Navi Mumbai, Maharashtra, India

Corresponding Author: Devendra P Patil, Department of Pedodontics and Preventive Dentistry, Terna Dental College, Navi Mumbai, Maharashtra, India, Phone: +91 9930372999, e-mail: pedotdc@gmail.com How to cite this article: Koticha P, Katge F, et al. Effectiveness of Virtual Reality Eyeglasses as a Distraction Aid to Reduce Anxiety among 6-10-year-old Children Undergoing Dental Extraction Procedure. Int J Clin Pediatr Dent 2019;12(4):297-302.

Source of support: Nil

Conflict of interest: None

Distraction is broadly classified into active and passive distraction techniques. ${ }^{11}$ Active forms promote the child's involvement into an activity during a dental procedure. These techniques involve several sensory components of the child. Some of the employed forms are interactive toys, singing songs, squeeze balls, controlled breathing, guided imaginary, and relaxation. Passive forms on other hand require the child to remain calm and quiet during a procedure. In this case, distraction is achieved by observation of the activity or stimulus rather than their overt participation. It includes auditory and audiovisual techniques. Several technological options are available for both visual and auditory distraction, such as background music, television sets, computer games, and virtual reality (VR) glasses. ${ }^{11,12}$

In recent years, there has been an increase in behavioral research in VR and virtual world. By encouraging a patient to focus 
his or her attention on other thoughts, less attention is available for the pain. Virtual reality utilizes advanced technologies to create virtual environments (VE) that allow patients to be immersed in an interactive, simulated world. These advanced systems interact at many levels with the $V E$, stimulating sights, sounds, and motion to encourage immersion in the virtual world to enhance distraction from pain. ${ }^{13,14}$ There have been very few studies of VR in a dental field. Some studies concluded that the use of audiovisual distraction is successful in decreasing not only anxiety ${ }^{15,16}$ but also pain perception. $^{3}$

Therefore, the aim of this study is to evaluate the effectiveness of VR eyeglasses as a distraction aid to reduce anxiety of children undergoing the dental extraction procedure.

\section{Materials and Methods}

\section{Subject Enrollment}

The approval to conduct the study was obtained from the Institutional Review Board (TDC/IRB-EC/137/2017). All procedures carried out in this study involving human participants were in accordance with the 1964 Helsinki declaration and its later amendments or comparable ethical standards as well as the International Conference on Harmonization guideline for good clinical practice. The sample size was calculated on a two-sample comparison of proportions of behaviors: one group with AV distraction and one control group. The inclusion of 30 patients $(n=60)$ with bilateral molars was considered sufficient to detect a statistically significant difference between two groups. The formula used for sample size calculation was $n=f(\alpha, \beta) \times[\sigma 2 /(\mu 1-\mu 2) 2]$, where $\sigma$ is the standard deviation (SD) of the within-subject differences $(\mu 1-\mu 2)$, and $f(\alpha, \beta)$ is a function of power and significance level of $5 \%(\alpha=0.05, \beta=$ 10.5 with $95 \%$ power).

The participants were selected from consecutive patients who presented for treatment to the Department of Pedodontics and Preventive Dentistry. Oral examination was done for every child using a mouth mirror and probe. Orthopantamograph (OPG) was advised to formulate the treatment plan. Full detailed treatment plans were explained to the parents. Written consents for treatment were obtained from the children's parents prior to the clinical procedures.

\section{Inclusion and Exclusion Criteria}

A total of 30 children with bilateral nonrestorable primary molars and those who demonstrated Frankl behavior rating 3-4 toward the dental procedures were included in this study.

Exclusion criteria for the study were medically compromised children, children with special health care needs, patients who had a bad experience with dentist, and Frankl behavior rating 1 and 2 .

\section{Study Design}

A total of 60 bilateral primary maxillary and mandibular molars in 30 patients aged 6-10 years were selected for this study. Bilateral nonrestorable primary molars were assigned for the dental extraction procedure. The randomization procedure was performed before the clinical procedure by using the split mouth study design. In a split mouth design, there are two study treatments (group I and group II) that are randomly assigned to left or right dentition sites. In group I (VR group), extraction was done with wearing VR eyeglasses whereas in group II (control group), the dental extraction procedure was done without wearing VR eyeglasses.
The treatment consisted of three consecutive sessions. All dental procedures were carried out by one pediatric dentist and one dental assistant at all three visits.

In first visit, all children in group I were introduced to VR eyeglasses using the tell-show-do technique and oral prophylaxis was done, whereas in group II all children had undergone the oral prophylaxis procedure only.

In second visit for group I, all children were given few minutes to get accustomed to the eyeglasses. The VR eyeglasses (BlackBug Virtual Reality Glasses 3D VR Box headsets for 4.7-6 inch mobile phones, model no: a236, India) used during extraction procedures blocked the visual field of the child completely and had in-built headphones to deliver the sound (Fig. 1). The device was connected to the mobile device (Apple iPhone 6, California) capable of playing MP4 audiovisual files (Fig. 1). A single episode of cartoon series "chotta bheem or doremon" was played for all subjects throughout the study. Once a VR device was secured on the child's eyes, playing the cartoon was started. Then, topical anesthetic agent (Lidayn topical aersol USP, India) was applied and necessary local anesthesia (Lignox 2\% with adrenaline 1:80,000, India) was administered, followed by extraction (Fig. 2). The technique employed for administration of local anesthesia in the maxillary

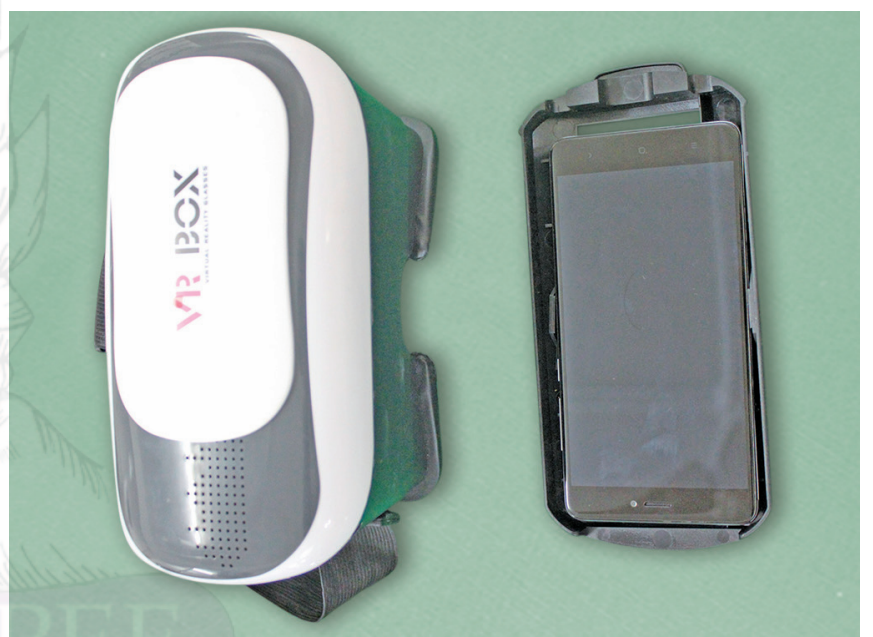

Fig. 1: BlackBug Virtual Reality Glasses 3D VR Box headsets for 4.7-6-inch mobile phones, model no. a236, India

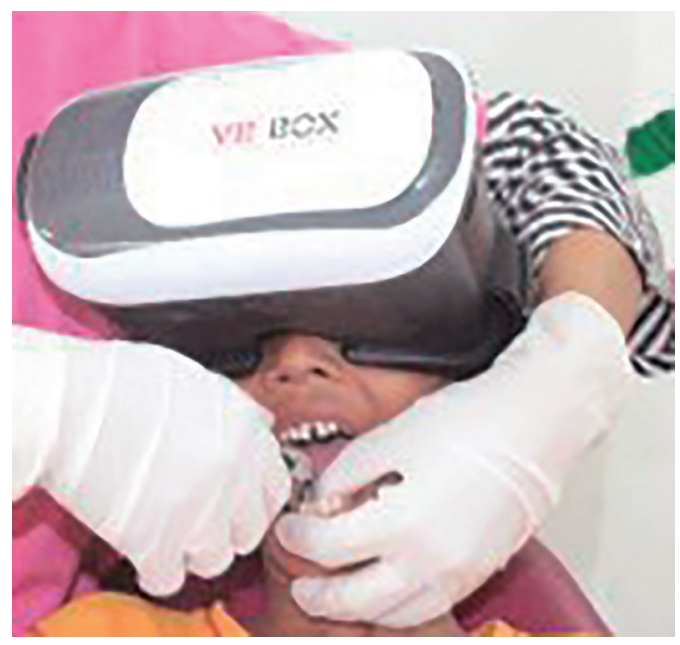

Fig. 2: Administration of local anesthesia followed by extraction of the tooth 


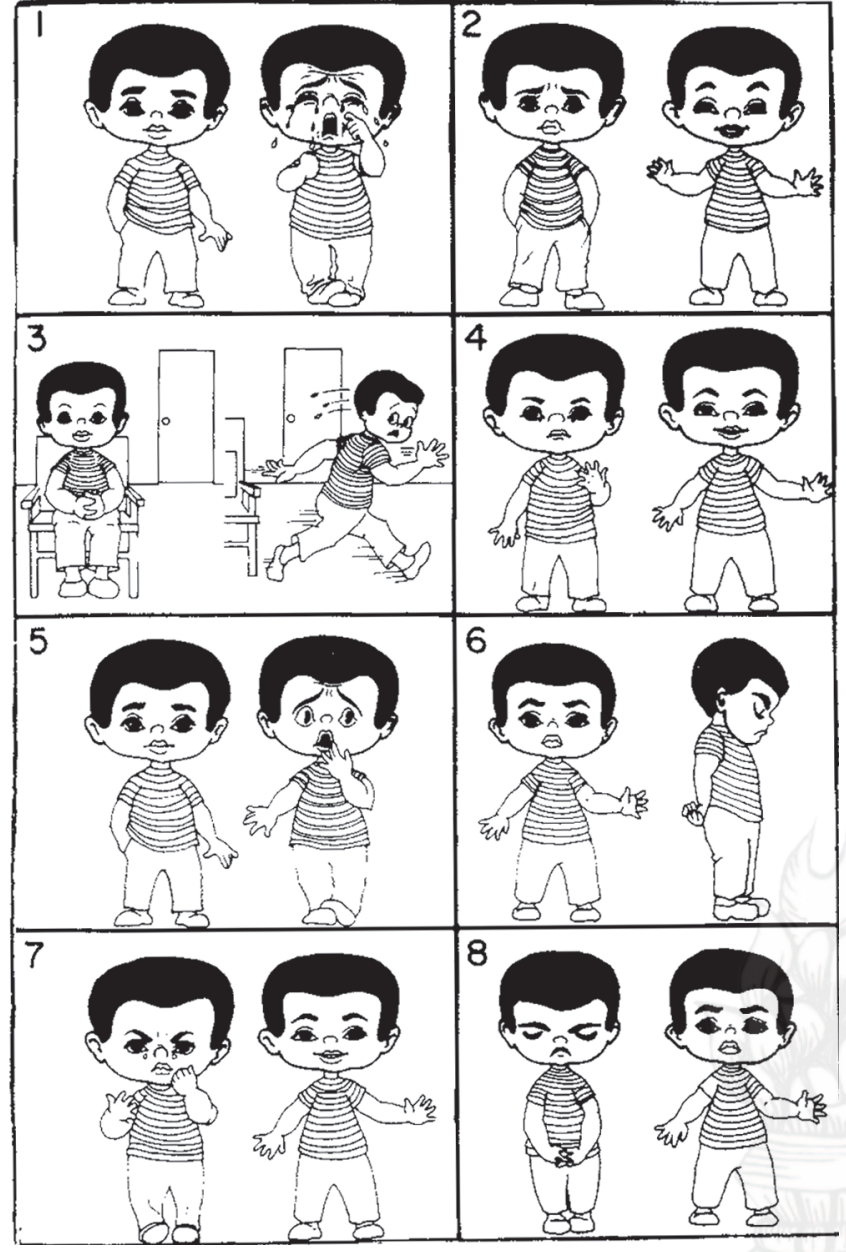

Fig. 3: Venham's picture test used for self-measured anxiety

arch was the infiltration technique, and the inferior alveolar nerve block technique was used in case of the lower arch.

In third appointment that took place 1-2 weeks after the second visit, extraction was performed in group II children without using VR eyeglasses. Each therapeutic session lasted about 30-45 minutes.

\section{Assessment of Anxiety}

Child's anxiety level in each procedural visit was assessed using a combination of three measures:

The Venham's picture test (VPT) (Fig. 3), which is a projective, psychometric, self-measure test that is used to measure the state anxiety of the young child. It comprises of eight cards, with two pictures in each card, one anxious figure and one nonanxious figure. The child was asked to select one of the figure he or she felt most like at that moment. All the cards were shown with number and ordered accordingly. If the child selects anxious figure, then score of 1 was recorded; if the child selects nonanxious figure, a score of 0 was recorded. The scale has a range of $0-8 .^{17}$

Pulse rate and oxygen saturation measured by the finger pulse oximeter (Choicemmed MD300C15D Pulse Oximeter, India) (Fig. 4), which is a direct measure of physiological arousal since its increase is attributed to stress during dental procedures. ${ }^{18}$

\section{Statistical Analysis}

The statistical analysis was performed using the SPSS version 17 software (SPSS Inc., Chicago, IL). Normality of the data was assessed

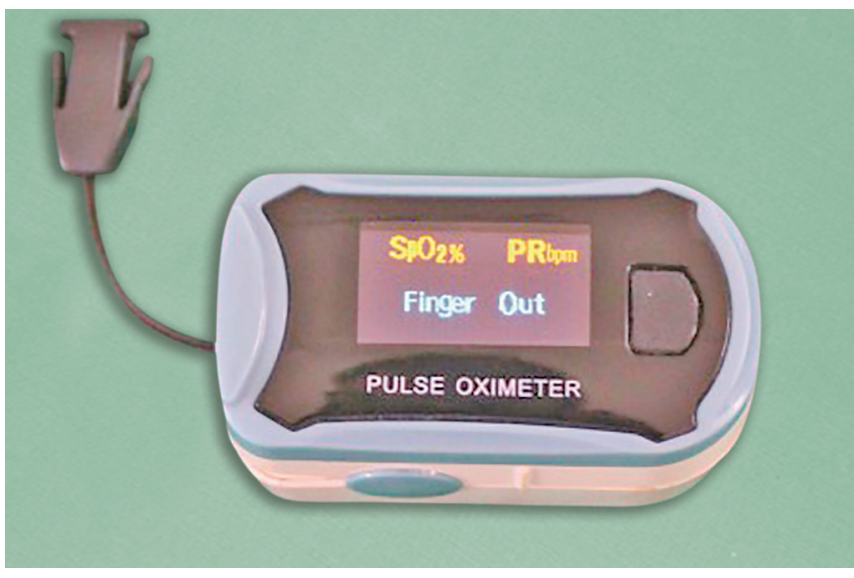

Fig. 4: Finger pulse oximeter (Choicemmed MD300C15D Pulse Oximeter, India) for measurement of pulse rate and oxygen saturation

using the paired $t$ test for comparison between the groups. Descriptive data are reported as frequencies, means, and SD. The significance level was set at $p<0.05$.

\section{Results}

A total of 30 patients $(n=60)$ with bilateral molars indicated for extraction were enrolled in the study. The age for the selected patients was in the range from 6 to 10 years. All patients reported for all three visits show $100 \%$ follow-up rate.

The scores in Table 1 showed and evaluated the changes in pulse rate, oxygen saturation, and the Venham's picture test. Table 1 depicts mean, SD, and standard error for different parameters in both groups.

The scores in Table 2 showed intergroup comparison of pulse rate, oxygen saturation, and self-reported anxiety using the Venham's picture test.

The mean pulse rate values before and after the extraction procedure in group I were $107.833 \pm 1.356$ and $108.4 \pm 0.927$, respectively. The difference between before and after pulse rate values in group I was found statistically nonsignificant $(p>0.05)$ as shown in Table 1. The mean pulse rate values before and after extraction in group II were $109.1 \pm 1.398$ and $112.233 \pm 0.774$, respectively. The difference between before and after pulse rate values in group II was found statistically significant $(p=0.03)$.

The mean oxygen saturation values before and after the extraction procedure in group I were $97.20 \pm 0.375$ and $96.83 \pm$ 1.801 , respectively. The difference between before and after oxygen saturation values in group I was found statistically nonsignificant $(p>0.05)$. The mean oxygen saturation values before and after the extraction procedure in group II were $96.933 \pm 0.2793$ and 96.933 \pm 0.2273 , respectively. The difference between before and after pulse rate values in group II was found statistically nonsignificant $(p>0.05)$ (Table 1).

The mean Venham's picture test values before and after the extraction procedure in group I were $2.9667 \pm 0.2815$ and $3.50 \pm 0.2815$, respectively. The difference between before and after Venham's picture test values in group I was found statistically significant $(p=0.03)$. The mean Venham's picture test values before and after the extraction procedure in group II were $3.60 \pm 0.327$ and $3.80 \pm 0.353$, respectively. The difference between before and after Venham's picture test values in group II was found statistically nonsignificant $(p>0.05)$ (Table 1). 
Effectiveness of Virtual Reality Eyeglasses as a Distraction Aid to Reduce Anxiety

Table 1: Vital signs and Venham's picture test (mean, SD, and significance) in both the groups before and after the extraction procedure

\begin{tabular}{|c|c|c|c|c|c|c|}
\hline Parameters & & & Mean & Standard deviation & $\begin{array}{l}\text { Standard error } \\
\text { mean }\end{array}$ & $\begin{array}{l}\text { Significance } \\
\text { ( } p \text { value) }\end{array}$ \\
\hline \multirow[t]{6}{*}{ Group I (VR group) } & Pulse & Before & 109.1000 & 7.66249 & 1.39897 & \\
\hline & & After & 112.2333 & 4.24007 & 0.77413 & 0.038 \\
\hline & Oxygen saturation & Before & 96.9333 & 1.52978 & 0.27930 & \\
\hline & & After & 96.9333 & 1.24522 & 0.22735 & 0.168 \\
\hline & Venham's picture test & Before & 3.6000 & 1.79271 & 0.32730 & \\
\hline & & After & 3.8000 & 1.93694 & 0.35363 & 0.934 \\
\hline \multirow{6}{*}{$\begin{array}{l}\text { Group II (control } \\
\text { group) }\end{array}$} & Pulse & Before & 107.8333 & 7.42820 & 1.35620 & \\
\hline & & After & 108.4000 & 5.08276 & 0.92798 & 0.223 \\
\hline & Oxygen saturation & Before & 97.2000 & 2.05779 & 0.37570 & \\
\hline & & After & 96.8333 & 1.80198 & 0.37570 & 0.350 \\
\hline & Venham's picture test & Before & 2.9667 & 1.54213 & 0.28155 & \\
\hline & & After & 3.5000 & 1.54213 & 0.28155 & 0.03 \\
\hline
\end{tabular}

Table 2: Intergroup comparison of pulse rate, oxygen saturation, and self-reported anxiety using Venham's picture test

\begin{tabular}{llcccc}
\hline Parameters & Groups & Mean & Std. deviation & Std. error mean & Sig. (two-tailed) \\
\hline Pulse & Group I & 108.4000 & 5.08276 & 0.92798 & 0.002 \\
& Group II & 112.2333 & 4.24007 & 0.77413 & 0.002 \\
Oxygen saturation & Group I & 96.8333 & 1.80198 & 0.32899 & 0.188 \\
& Group II & 97.3667 & 1.24522 & 0.22735 & 0.188 \\
Venham's picture test & Group I & 3.5000 & 1.87083 & 0.34157 & 0.544 \\
& Group II & 3.8000 & 101.93694 & 0.35363 & 0.544 \\
\hline
\end{tabular}

In intergroup comparison for pulse rate (Table 2), group I (108.4 \pm 0.927$)$ showed lesser pulse rate values as compared to group II (112.233 \pm 0.774$)$, which was statistically significant $(p<0.05)$. The oxygen saturation and the Venham's picture test showed no significant difference with any of two groups ( $p>0.05)$.

\section{Discussion}

Dental procedures, local anesthesia administration in particular, are often a source of anxiety and stress in children, in turn an increase in pain reactivity. Therefore, it is very important to use specific interventions to distract them from dental treatment procedures. Distraction, one of the psychobehavioral approaches used in medical and dental treatment situations, is defined as a nonaversive approach used to modify a child's discomfort by disrupting his or her attention away from the main task to accomplish successful treatment with high quality. 19,20

This study thus tested the effectiveness of distraction using VR eyeglasses on anxiety levels in children undergoing the dental extraction procedure. The present study was designed as a splitmouth study design. The design is characterized by subdividing the child's dentition into halves (right and left), where two different treatment modalities are assigned to one side randomly, in order to allow further outcome evaluation. Each participant acts as their own control by making within-patient rather than betweenpatient comparisons, thus diminishing intersubject variability and increasing study accuracy and power. ${ }^{21}$

It has been demonstrated that distraction techniques are less effective in individuals who have a previous bitter pain experience. ${ }^{22}$ Therefore, in the present study subjects were excluded if they had previous medical or dental history.

Anxiety was measured by both objective and subjective means. The subjective measure was done by the Venham's picture test. The objective measure of anxiety was assessed by measuring pulse rate and oxygen saturation with the help of a pulse oximeter. The pulse rate is governed by the nervous system, especially the autonomic nervous system (ANS) that reflects negative emotions in terms of physiological parameters such as heart rate, respirations, and body temperature. Therefore, physiological responses of the ANS are indicators used to tell if a person is under stress or relaxation. $^{18,23}$

The mean pulse rate value in group II after extraction $(112.233 \pm$ 0.774 ) was greater than mean pulse rate value before extraction $(109.1 \pm 1.398)$, which was statistically significant. If the pulse rate value increases after the dental procedure relatively, it gives an idea that the child is anxious. This suggests pulse rate as an important parameter for assessing anxiety in children. The importance of pulse rate as a physiological parameter of anxiety in children was first reported by Mccarthy. ${ }^{24}$ In the present study, in intergroup comparison, VR eyeglasses showed their effectiveness in respect to pulse rate. These results were in accordance with previous studies conducted by Prabhakar et al., ${ }^{9}$ Hoffman et al., ${ }^{25}$ and Nilson et al. ${ }^{26}$ They concluded that the use of audiovisual distraction during dental treatment was more effective in managing anxious children than using audio distraction solely. ${ }^{9,25,26}$ These benefits may be related to more immersive images due to the occlusive headsets that project the images right in front of the eyes of the user and block out realworld stimuli. The child's attention is focused on what happen in the virtual world rather than on the surrounding environment. ${ }^{7}$ The application of VR distraction is based on the assumption that pain perception has a large psychological component and that pain attracts a strong attentive response causing anxiety. ${ }^{27}$ Recently, it has also been found that VR changes the way people interpret incoming pain signals and actually reduces the amount of painrelated brain activity. ${ }^{28}$ 
The changes in oxygen saturation in groups, group I $(97.20 \pm$ 0.375 and $96.83 \pm 1.801)$ and group II $(96.933 \pm 0.2793$ and $96.933 \pm$ $0.2273)$, before and after the extraction procedure were observed when VR eyeglasses were used. These changes observed were statistically nonsignificant. Similar results were shown by Prabhakar et al. ${ }^{9}$ and Kaur et al. ${ }^{29}$ who concluded that audiovisual distraction certainly reduces the pain perception and distress in children during intravenous injection. They also concluded that $\mathrm{AV}$ distraction was more effective in managing anxious pediatric patients in terms of heart rate and oxygen saturation level. In intergroup comparison, no statistical difference was observed in anxiety level in children. Although oxygen saturation is not an immediate physiological measure of anxiety, analysis was done using this parameter too as there are no studies carried out to assess the correlation between both the factors. Additionally, the pulse oximeter used in the study had this inherent parameter. ${ }^{30}$

The Venham's picture test used in the present study showed significant values with group I before $(2.9667 \pm 0.2815)$ and after $(3.50 \pm 0.2815)$ the extraction procedure wearing the VR eyeglasses. These results were in accordance to similar studies conducted by Sullivan et al., ${ }^{6}$ Prabhakar et al., ${ }^{9}$ Hoge et al., ${ }^{16}$ and Guinot et al. ${ }^{31}$ They found no significant differences in the measures of anxiety obtained with the Venham's picture test. The results of the present study were contradicting with the study conducted by Baghdadi. ${ }^{32}$ They found a significance difference in the Venham's picture test during dental treatment between the audio distraction technique and control situation. ${ }^{31}$

\section{Limitation of the Study}

The initial limitation of the present study was smaller sample size. A larger sample size in a general clinical setting might have cleared the differences in the use of AV distraction due to anxiety and behavior measures. The other limitation to the study was the design of the eyeglass system (BlackBug Virtual Reality Glasses). This design allows a slight visual access to the surrounding environment. Hence, the patients were not distracted from procedures performed in the oral cavity. However, in surrounding environment sound and visual field of the children could not be blocked due to oversized eyeglasses and earphones.

\section{Conclusion}

The present study confirms the efficacy of VR distraction as a means of the behavior management technique in our day-to-day practice.

The use of VR as a distraction technique improves the physiologic parameter of children aged 6-10 years but does not reduce the patient's self-reported anxiety according to Venham's picture test used.

The anxiety-inducing appearance of dental equipment and the child's focusing on all details of the procedure is one of the most important reasons for stress associated with dental procedures in children. Therefore, the positive effects of VR distraction on anxiety in children in the present study are attributed to blockage of children's visual fields and as a result to a successful distraction technique.

In our era of digital dentistry, use of an audiovisual aid can be an adjunct reducing the anxiety of children.

\section{Clinical Significance}

In today's digitalized world, children are attracted to digital games, cartoon series, mobiles, digital tablets, etc. Using VR eyeglasses as an audiovisual distraction is useful in managing anxiety in fearful children during dental treatments. This technique of distraction also reduces operatory stress on the pediatric dentist. Hence, this technique can be utilized in routine pediatric practice as an adjunct to other behavior management techniques.

\section{ACKNOWLedgment}

We would like to thank Dr Nikhil Bhanushali of the Department of Public Health Dentistry for his assistance with the statistical analysis for the study.

\section{References}

1. Bankole $\mathrm{OO}$, Aderinokun GA, et al. Maternal and child's anxietyeffect on child's behaviour at dental appointments and treatments. Afr J Med Med Sci 2002;31:349-352.

2. Folayan MO, Fatusi A. Effect of psychological management techniques on specific item score change during the management of dental fear in children. J Clin Pediatr Dent 2005;29:335-340. DOI: 10.17796/jcpd.29.4.d431t7024u4037u6.

3. Asl Aminabadi $N$, Erfanparast $L$, et al. The impact of virtual reality distraction on pain and anxiety during dental treatment in 4-6 yearold children: a randomized controlled clinical trial. J Dent Res Dent Clin Dent Prospects 2012;6:117-124. DOI: 10.5681/joddd.2012.025.

4. Richmond BJ, Sato T. Enhancement of inferior temporal neurons during visual discrimination. J Neurophysiol 1987;58:1292-1306. DOI: 10.1152/jn.1987.58.6.1292.

5. Kleiber C, Harper DC. Effects of distraction on children's pain and distress during medical procedures: a meta-analysis. Nurs Res 1999 Jan-Feb;48(1):44-49. DOI: 10.1097/00006199-199901000-00007.

6. Sullivan C, Schneider PE, et al. The effect of virtual reality during dental treatment on child anxiety and behavior. ASDC J Dent Child 2000;67:193.

7. Wismeijer AA, Vingerhoets AJ. The use of virtual reality and audiovisual eyeglass systems as adjunct analgesic techniques: A review of the literature. Ann Behav Med 2005;30:268-278. DOI: 10.1207/s15324796abm3003_11.

8. Sinha M, Christopher NC, et al. Evaluation of nonpharmacologic methods of pain and anxiety management for laceration repair in the pediatric emergency department. Pediatrics 2006;117:1162-1168. DOI: 10.1542/peds.2005-1100.

9. Prabhakar AR, Marwah N, et al. A comparison between audio and audiovisual distraction techniques in managing anxious pediatric dental patients. J Indian Soc Pedod Prev Dent 2007;25:177-182. DOI: 10.4103/0970-4388.37014.

10. Dahlquist LM, Weiss KE, et al. Effects of videogame distraction using a virtual reality type head-mounted display helmet on cold pressor pain in children. J Pediatr Psychol 2009;34:574-584. DOI: 10.1093/ jpepsy/jsn023.

11. Armfield JM, Spencer AJ, et al. Dental fear in Australia: who's afraid of the dentist? Aust Dent J 2006;51:78-85. DOI: 10.1111/j.1834-7819.2006. tb00405.x.

12. Abdelmoniem SA, Mahmoud SA. Comparative evaluation of passive, active, and passive-active distraction techniques on pain perception during local anesthesia administration in children. J Adv Res 2016;7(3):551-556. DOI: 10.1016/j.jare.2015.10.001.

13. Wiederhold MD, Wiederhold BK. Virtual reality and interactive simulation for pain distraction. Pain Medicine 2007;8(3):S182-S188. DOI: 10.1111/j.1526-4637.2007.00381.x.

14. Wiederhold MD, Gao K, et al. Clinical use of virtual reality distraction system to reduce anxiety and pain in dental procedures. Cyberpsychol Behav Soc Netw 2014;17(6):359-365. DOI: 10.1089/cyber. 2014.0203.

15. El-Sharkawi HF, El-Housseiny AA, et al. Effectiveness of new distraction technique on pain associated with injection of local anesthesia for children. Pediatr Dent 2012;34(2):35-38. 
16. Hoge MA, Howard MR, et al. Use of video eyewear to manage distress in children during restorative dental treatment. Pediatr Dent 2012;34(5):378-382.

17. Venham LL, Gaulin-Kremer E, et al. Interval rating scales for children's dental anxiety and uncooperative behavior. Paediatr Dent 1980;2(3):195-202.

18. Navit S, Johri N, et al. Effectiveness and Comparison of Various Audio Distraction Aids in Management of Anxious Dental Paediatric Patients. J Clin Diagn Res 2015;9(12):ZC05-ZC09. DOI: 10.7860/ JCDR/2015/15564.6910.

19. Pinkham JR. Behavior management of children in the dental office. Dent Clin North Am 2000;44(3):471-486.

20. Al-Khotani A, Bello LA, et al. Effects of audiovisual distraction on children's behaviour during dental treatment: a randomized controlled clinical trial. Acta Odontol Scand 2016;74(6):494-501. DOI: 10.1080/00016357.2016.1206211.

21. Pozos-Guillén $A$, Chavarría-Bolaños $D$, et al. Split-mouth design in Paediatric Dentistry clinical trials. Eur J Paediatr Dent 2017 Mar;18(1):61-65. DOI: 10.23804/ejpd.2017.18.01.13.

22. Slifer $\mathrm{KJ}$, Tucker $\mathrm{CL}$, et al. Helping children and caregivers cope with repeated invasive procedures: how are we doing? J Clin Psychol 2002;9(2):131-152.

23. Kreibig SD. Autonomic nervous system activity in emotions: a review. Biol Psychol 2010;84(3):394-421. DOI: 10.1016/j.biopsycho. 2010.03.010.

24. Mccarthy F. A clinical study of blood pressure responses to epinephrine-containing local anesthetic solutions. J Dent Res 1957; 36(1):132-141. DOI: 10.1177/00220345570360010901.
25. Hoffman $\mathrm{H}$, Garcia-Palacios A, et al. The effectiveness of virtual reality for dental pain control. Cyberpsychol Behav 2001;4(4):527-535. DOI: 10.1089/109493101750527088.

26. Nilson S, Finnstrom B, et al. The use of virtual reality for needle related procedural pain and distress in children and adolescents in a pediatric oncology unit. Eur J Oncol Nurs 2009;13(2):102-109. DOI: 10.1016/ j.ejon.2009.01.003.

27. Sharar SR, Carrougher GJ, et al. Factors influencing the efficacy of virtual reality distraction analgesia during postburn physical therapy: preliminary results from 3 ongoing studies. Arc Phys Med Rehabil 2007;88(12):S43-S49. DOI: 10.1016/j.apmr.2007.09.004.

28. Hoffman HG, Patterson DR, et al. Effectiveness of virtual reality-based pain control with multiple treatments. Clin J Pain 2001;17(3):229-235. DOI: 10.1097/00002508-200109000-00007.

29. Kaur B, Sarin J, et al. Effectiveness of cartoon distraction on pain perception and distress in children during intravenous injection. IOSR Journal of Nursing and Health Science 2014;3(3):8-15. DOI: 10.9790/1959-03320815.

30. Rayen R, Muthu MS, et al. Evaluation of physiological and behavioral measures in relation to dental anxiety during sequential dental visits in children. Indian J Dent Res 2006;17(1):27-34. DOI: 10.4103/09709290.29895 .

31. Guinot Jimeno F, Mercadé Bellido $M$, et al. Effect of audiovisual distraction on children's behavior anxiety and pain in the dental setting. Eur J Paediatr Dent 2014;15(3):297-302.

32. Baghdadi ZD. Evaluation of audio analgesia for restorative care in children treated using electronic Dental anesthesia. J Clin Pediatr Dent 2000;25(1):9-12. 